\title{
Cutaneous Nodules as an Initial Manifestation of Neuroendocrine Carcinoma with Unknown Primary Site: A Case Report
}

\author{
(1) Mahmut Esat Tanrıbilir ${ }^{1}$, (1) Ceren Bilkan Öge², (1) Esra Adıșen ${ }^{1}$ \\ ${ }^{1}$ Gazi University Faculty of Medicine, Department of Dermatology, Ankara, Turkey \\ ${ }^{2}$ Gazi University Faculty of Medicine, Department of Pathology, Ankara, Turkey
}

\section{ABSTRACT}

Neuroendocrine tumors are heterogenous group of neoplasms arising from cells of neuroendocrine origin. They have a wide spectrum of clinical behavior and malignant potential. Cutaneous metastases of these tumors have been reported very rarely. We report a patient diagnosed with metastatic neuroendocrine carcinoma after a biopsy was performed from his skin nodules. Cutaneous nodules were one of the first manifestations of his internal malignancy and helped us to diagnose and manage the patient.

Keywords: Neuroendocrine tumor, Cutaneous metastasis, Cancer of unknown primary

\section{Introduction}

Neuroendocrine tumors are rare neoplasms arising from cells of neuroendocrine origin. These tumors commonly originate from gastrointestinal tract, pancreas, lung, thymus and other endocrine organs [1]. They most commonly metastasize to lymph nodes, liver and lung [2]. However, cutaneous metastases of these tumors are very rare. In this article, we present a patient diagnosed with neuroendocrine carcinoma after his cutaneous nodules appeared. His cutaneous findings were one the first manifestations of his underlying malignancy.

\section{Case Report}

A 72-year old man visited our clinic with multiple nodules on his trunk. He had a history of jaundice, malaise and weight loss for 3 weeks. His cutaneous nodules first appeared on right subclavicular area, followed by 4 new nodules on trunk within 1 week. Nodules were rapidly enlarging, but he did not describe any symptoms. He was brought to emergency department with intractable jaundice and constitutional symptoms a few days ago. Hepatobiliary ultrasonography was performed. In the head of pancreas; $2.5-\mathrm{cm}$ sized, hypoechoic mass containing cystic, necrotic areas was observed.

The patient was directed to oncology department and malignancy work-up has been started. He was consulted to our dermatology department for diagnosis of cutaneous nodules. In the dermatologic examination; painless, firm, dome-shaped, red-purplish five discrete nodules were present on right subclavicular area, abdomen and right lateral site of thorax (Figure 1, 2). A biopsy was taken from the lesions, monomorphic, atypical small cells with round, hyperchromatic nuclei were seen in subcutaneous tissue with hematoxylin and eosin staining (Figure 3). Tumor cells were stained positive with chromogranin, synaptophysin, CD56 and thyroid transcription factor-1 (TTF-1) (Figure 4, 5); but negative with CK7, CK20, CD45, CDX2. Immunohistochemical staining features of cells were compatible with metastatic neuroendocrine carcinoma. Ki-67 proliferation index was studied and more than 95\% proliferative activity was reported (Figure 6).

Further diagnostic tests were initiated to detect the primary tumor. In his thoracoabdominal computed tomography $(\mathrm{CT}) ; 12 \times 10 \mathrm{~cm}$ 


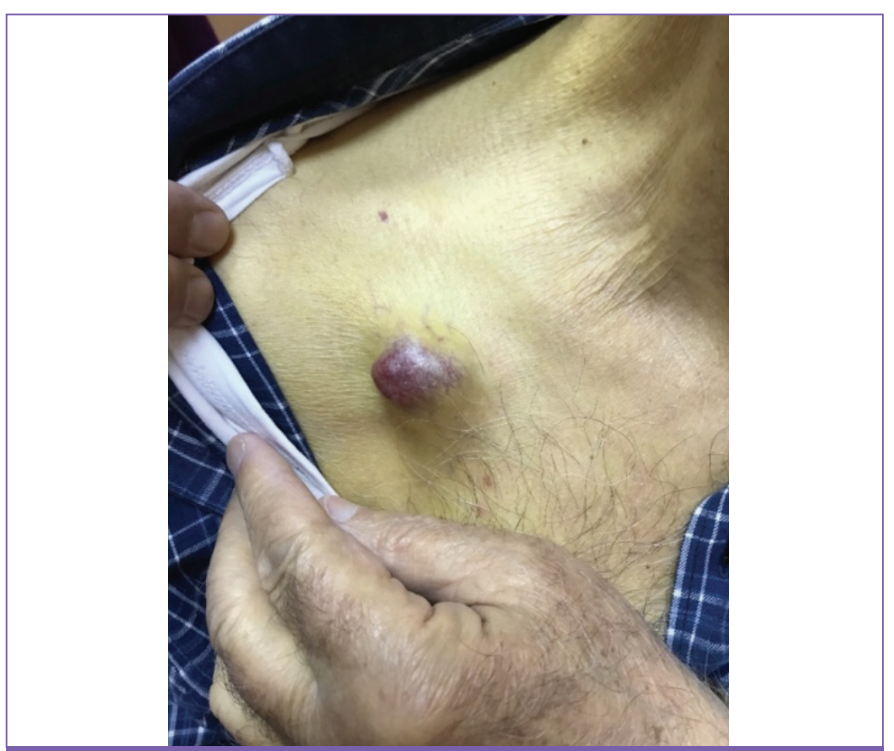

Figure 1. A firm, painless, 3×2-cm sized, purple nodule on the right subclavicular area

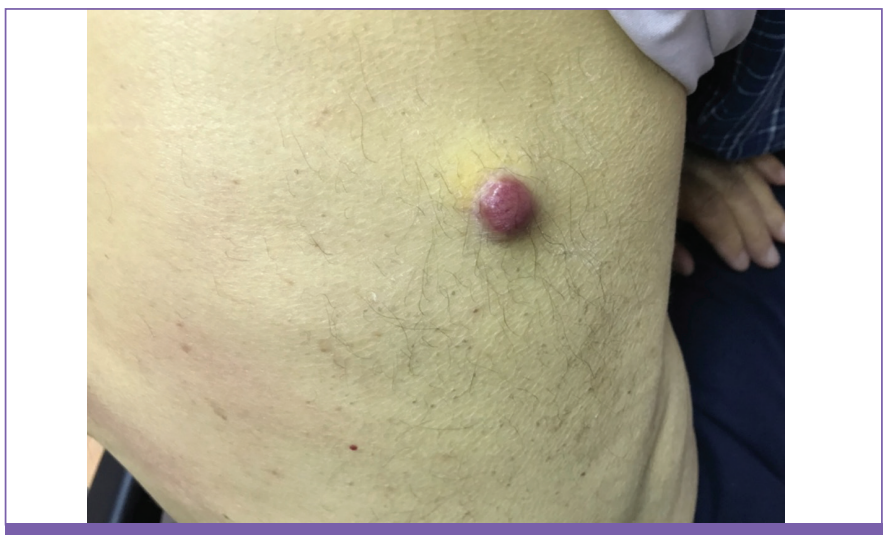

Figure 2. A painless, smooth-contoured, round-shaped, 2x2$\mathrm{cm}$ sized red-purple nodule on the right lateral site of thorax

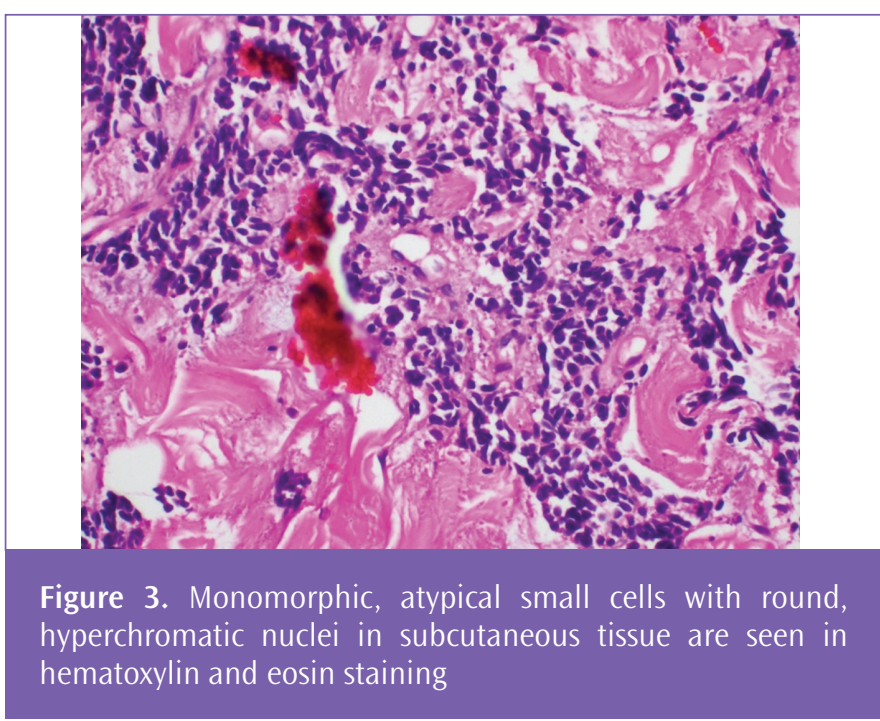

sized, lobulated mass containing cystic and necrotic areas was observed in the right lung parenchyma. The mass was obliterating right main bronchus and invading right middle and inferior lobe arteries. Tumoral masses were also observed in head and neck of pancreas and adrenal glands in CT. Thoracentesis was performed, but atypical cells were not observed. Endoscopic retrograde

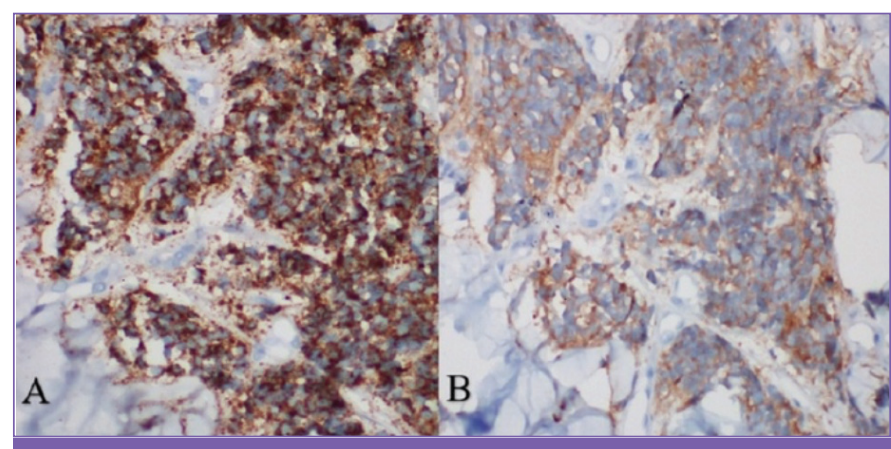

Figure 4. Immunohistochemical features of tumor cells. Tumor cells are immunoreactive with (A) chromogranin and (B) synaptophysin

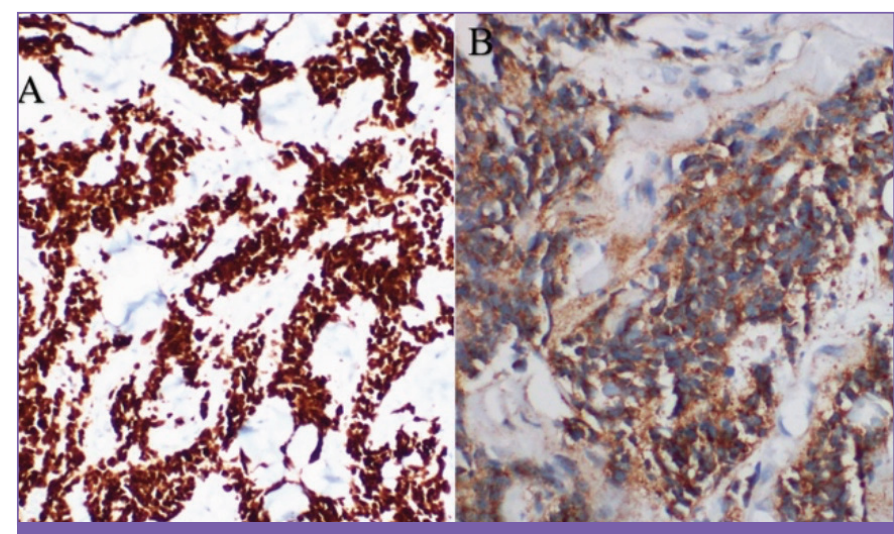

Figure 5. Immunohistochemical features of tumor cells. Tumor cells are immunoreactive with (A) thyroid transcription factor-1 (SP141 clone) and (B) CD56

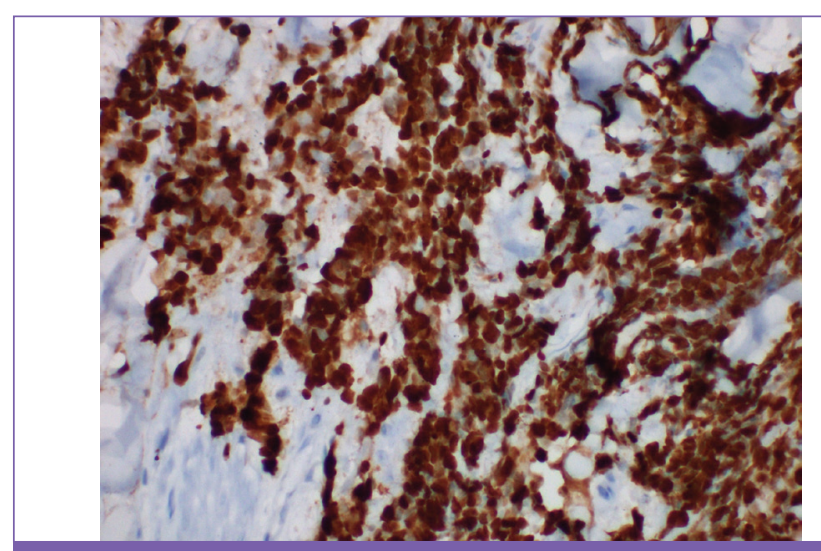

Figure 6. Tumor cells are stained diffusely positive with Ki-67 
cholangiopancreatography (ERCP) procedure was performed by gastroenterologists. During ERCP, a biopsy was taken from ampulla of Vater; but neuroendocrine tumor or a tumor with epithelial origin could not be detected histopathologically. The exact location of primary tumor was still being investigated; etoposide/cisplatin chemotherapy has been started for metastatic neuroendocrine tumor.

\section{Discussion}

Neuroendocrine tumors consist of heterogenous group of neoplasia originating from neuroendocrine cells throughout the body. They most commonly arise from gastrointestinal tract and bronchopulmonary system [3]. Their clinical presentation, tumor biology and metastatic potential vary widely. Different from their common metastatic sites-liver, lung or lymph nodes; cutaneous metastases of these tumors are very rare. Up to date, less than 40 cases with neuroendocrine tumor metastases to skin were reported [4]. Five of them had no systemic symptoms, presented only with cutaneous findings [4].

Contrary to patients who were diagnosed with neuroendocrine tumor and cutaneous findings arising later during their followup, or asymptomatic patients presenting only with cutaneous findings who were diagnosed with neuroendocrine tumor later; our patient's systemic and cutaneous findings emerged simultaneously. Dermatologic examination and histopathologic evaluation of his skin nodules played an important role in the diagnosis and management of the patient.

Merkel cell carcinoma, highly aggressive primary neuroendocrine carcinoma of skin, should be distinguished from metastatic neuroendocrine tumors. CK20 positivity and TTF-1 negativity are important features of Merkel cell carcinoma [5]. In our patient, CK20 staining was negative but TTF-1 staining was positive. His immunohistochemical and radiological findings, together with clinical signs and symptoms, were consistent with metastatic neuroendocrine tumor rather than Merkel cell carcinoma.

Cutaneous manifestations of neuroendocrine tumor metastases vary. Single or multiple, non-ulcerated, painless nodules are the most common manifestation [6]. Rarely, painful metastatic lesions were reported in the literature [7]. Our patient presented with multiple, painless, firm, red-purplish nodules. His systemic symptoms occurred 1 week after his skin nodules. Considering his systemic symptoms and imaging findings, underlying systemic malignancy was likely. His nodules became a practical location for biopsy and diagnosis of neuroendocrine carcinoma was confirmed. This case report is a rare example of neuroendocrine tumor metastasis to skin.
Neuroendocrine tumors rarely metastasize to skin, but their cutaneous findings might be the first manifestation of underlying malignancy. Clinical suspicion and histopathologic examination are important for these rare tumors. Dermatologists play important role in such cases and multidisciplinary approach is important in the management of these patients.

\section{Ethics}

Informed Consent: Informed consent was filled out by all participants.

Peer-review: Externally and internally peer-reviewed.

\section{Authorship Contributions}

Surgical and Medical Practices: M.E.T., Concept: M.E.T., Design: M.E.T., E.A., Data Collection or Processing: M.E.T., C.B.Ö., Analysis or Interpretation: M.E.T., C.B.Ö., E.A., Literature Search: M.E.T., C.B.Ö., E.A., Writing: M.E.T., C.B.Ö., E.A.

Conflict of Interest: No conflict of interest was declared by the authors.

Financial Disclosure: The authors declared that this study received no financial support.

\section{References}

1. Yalcin, S. Introduction to Neuroendocrine Tumours. In Öberg K, Yalcin $\mathrm{S}$, editors. Neuroendocrine Tumours Diagnosis and Management. Springer;2015.p.1.

2. Modlin IM, Sandor A. An analysis of 8305 cases of carcinoid tumors. Cancer 1997;79:813-829.

3. Salyers WJ, Vega KJ, Munoz JC, Trotman BW, Tanev SS. Neuroendocrine tumors of the gastrointestinal tract: Case reports and literature review. World J Gastrointest Oncol 2014;6:301-310.

4. Jedrych J, Busam K, Klimstra DS, Pulitzer M. Cutaneous metastases as an initial manifestation of visceral well-differentiated neuroendocrine tumor: a report of four cases and a review of literature. J Cutan Pathol 2014;41:113122.

5. Kervarrec T, Tallet A, Miquelestorena-Standley E, Houben R, Schrama D, Gambichler T, Berthon P, Le Corre Y, Hainaut-Wierzbicka E, Aubin F, Bens G, Tabareau-Delalande F, Beneton N, Fromont G, Arbion F, Leteurtre E, Touzé A, Samimi M, Guyétant S. Diagnostic accuracy of a panel of immunohistochemical and molecular markers to distinguish Merkel cell carcinoma from other neuroendocrine carcinomas. Mod Pathol 2019;32:499510 .

6. Amorim GM, Quintella D, Cuzzi T, Rodrigues R, Ramos-E-Silva M. Cutaneous Metastasis of Neuroendocrine Carcinoma with Unknown Primary Site: Case Report and Review of the Literature. Case Rep Dermatol 2015;7:263-274.

7. Donati P, Panetta C, Cota C, Paolino G, Muscardin L. Carcinoid metastasis of the skin appearing as painful tumor. J Dermatol 2013;40:415-417. 